\title{
DUST IN THE MAGELLANIC CLOUDS
}

\author{
PAUL HODGE \\ University of Washington \\ Dept. of Astronomy, FM-20 \\ Seattle, Washington 98195 \\ U.S.A.
}

\begin{abstract}
The dust content of the Magellanic Clouds can be studied using optical, ultraviolet, infrared and, indirectly, radio wavelength data. All recent studies show that the dust content is lower than that of the Milky Way Galaxy for both Clouds and that the optical properties of the dust are different. At ultraviolet wavelengths, the $2165 \AA$ "bump" in the extinction curve is significantly smaller than in the Galaxy (this now appears NOT to be a consequence of the lower heavy element abundances) and the far ultraviolet (shortward of $\sim 2000 \AA$ ) extinction is greater than in the Galaxy (this IS likely to be a consequence of the lower heavy element abundances). New optical data on background galaxies suggest that the total extinction in the central parts of both the LMC and the SMC is approximately 1.5 magnitudes. High local extinction values are derived from uv and optical observations of star-forming regions, where a spatial correlation with CO detections is sometimes, but not always, found.
\end{abstract}

\section{Methods of Study}

The dust content of the Magellanic Clouds has been the subject of a wide variety of types of study in recent years. This paper concentrates on optical techniques, but mention is made here of some representative references to papers exploiting other methods. For instance, Boggs (1990) has shown that ultraviolet studies of MC stars, combined with new analysis of our Galaxy's outer regions (where abundances are nearly comparable with the MC's low heavy element abundances) indicate that the ultraviolet extinction shortward of $\sim 2000 \AA$ shows an excess, compared to the solar neighbourhood, that is correlated with abundance. The anomalously small $2165 \AA$ "bump" on the other hand is not abundance-dependent. Other ultraviolet studies of MC extinction have been published by Koomneef and Code (1981), Nandy $e t$ al. (1981), Hutchings (1982), Lequeux et al. (1984), Fitzpatrick (1985), Clayton and Martin (1985) and many others. The infrared extinction and emission due to dust in the MCs has been discussed by Morgan and Nandy (1982), Koomneef 
(1982), Clayton and Martin (1985), Schwering (1988) and many others. A much longer history of the optical extinction exists. I mention here only the large compilation of reddenings published by Isserstedt (1975), the study by Greve et al. (1990) of the dust in LMC emission nebulae, and the results of the multiwavelength study of extinction in very young clusters carried out by Lee (1990).

The methods of study mentioned in the above paragraph are all relatively straight-forward, traditional techniques, for which accuracies are well-defined and results are easily evaluated. The remainder of this paper deals with two methods, both optical, which are comparatively difficult to carry out, more awkward to describe, and nearly impossible to evaluate.

\section{Background Galaxy Counts}

\subsection{THE SMC}

The rather chequered history of the study of the total extinction i the SMC using background galaxy counts has been outlined in Hodge (1974a). The main problems with this kind of endeavour were outlined there and in Wesselink (1961) and MacGillivray (1975); three errors can seriously affect the results : (a) counting efficiency depends on the star density and the dependence is different for different persons or machines, (b) at faint levels, faint pairs or groups of stars can be mistakenly thought to be galaxy images by the eye or machine, and (c) other non-stellar objects in the MCs, such as emission nebulae, can be counted erroneously. The references cited give the various ways by which attempts have been made to overcome these difficulties, but the results of even the most recent analyses remain somewhat tentative because of them.

Figure 1 shows the results found in Hodge's (1974a) study, based on counts made by eye on both Schmidt and large reflector plates. The dust was found to be detectable out to distances from the core of the SMC as large as 4 degrees and the central peak of extinction, located in the high star-density area at the southern end of the bar, was found to cause 1.3 magnitudes of extinction in $\mathrm{V}$.

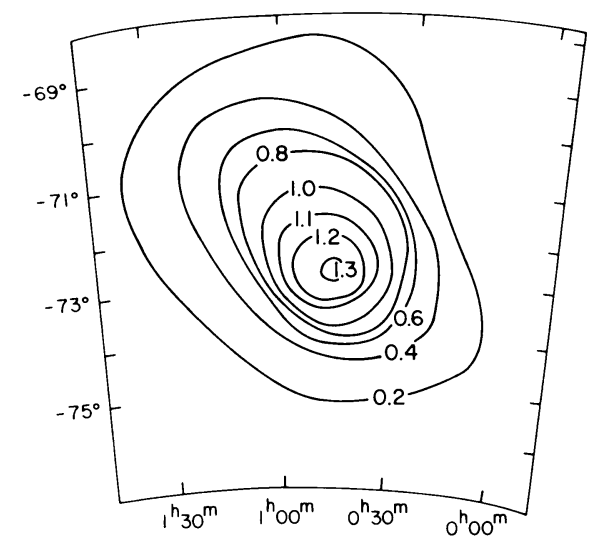

Figure 1. Smoothed distribution of extinction due to dust in the SMC. Units are V magnitudes (from Hodge 1974a). 


\subsection{THE LMC}

No one has carried out a complete survey of background galaxies for the LMC, probably because of its very large angular extent and the large number of emission nebulae and faint star clusters that could cause confusion on smallscale (e.g., Schmidt) plates. Aside from as yet unpublished attempts to use special software with automated counts (e.g., MacGillivray 1990), the only attempt to measure the extinction through the LMC is that of Gurwell and Hodge (1990), who used 4-m plates of selected regions, varying in distance from the centre of the Cloud from 1 degree to 7.3 degrees. They carried out several tests of the counting efficiency of their results to allow correction for the various kinds of errors listed in Section 2.0 above; these consisted primarily in repeating counts with "plate sandwiches", in which two plates were placed emulsion to emulsion, and the effects of varying the star foreground density could be isolated.

Figure 2 illustrates the results of the background galaxy counts for the LMC. A large decrease is found in the corrected galaxy densities toward the central areas of the Cloud. The effects of extinction seem to be detectable out to approximately 6 degrees from the centre. Assuming a uniform spatial density for galaxies in this part of the sky, one can calculate that the maximum general extinction near the centre of the LMC is 1.5 magnitudes in V. This value agrees well with the extinction found for the most reddened stars (Isserstedt 1975) and for the Cepheids that depart the most from the PL relation (Payne-Gaposchkin and Gaposchkin 1966).

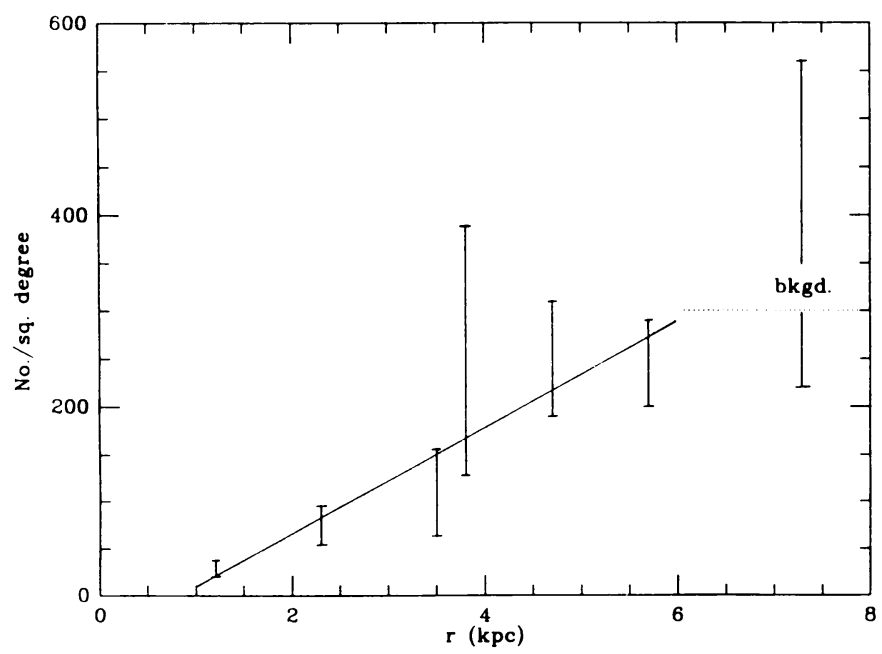

Figure 2. The density of detected galaxies behind the LMC, plotted against the radial distance from the centre of the LMC. 


\section{Discrete Dust Clouds}

\subsection{THE LMC}

The discrete dust clouds of the SMC, traditionally referred to as "dark nebulae", have been catalogued on the basis of a global search on Schmidt plates by Hodge (1974b) and by van den Bergh (1974). A more recent survey based on 4-m plates has been completed by Hodge (1990). All papers agree that the SMC dark nebulae are smaller than those of the LMC (Section 3.2), with mean dimensions from the Schmidt plate survey of $42 \mathrm{pc}$. The sizes range from 20 $\mathrm{pc}$ to $120 \mathrm{pc}$, with the lower limit likely to be an artifact of the search procedure and plate material. These papers point out the highly subjective nature of these searches and find, therefore, that the agreement from the different searchers and the different plate material is both surprising and reassuring. In evaluating and using these catalogues it is important to keep in mind the fact that the dust clouds can only be detected in front of a relatively smooth background luminosity, and that the searches are therefore not of uniform efficiency across the image of the SMC; in particularly empty areas, as well as in very "grainy" areas, the likelihood of detecting dark nebulae is small.

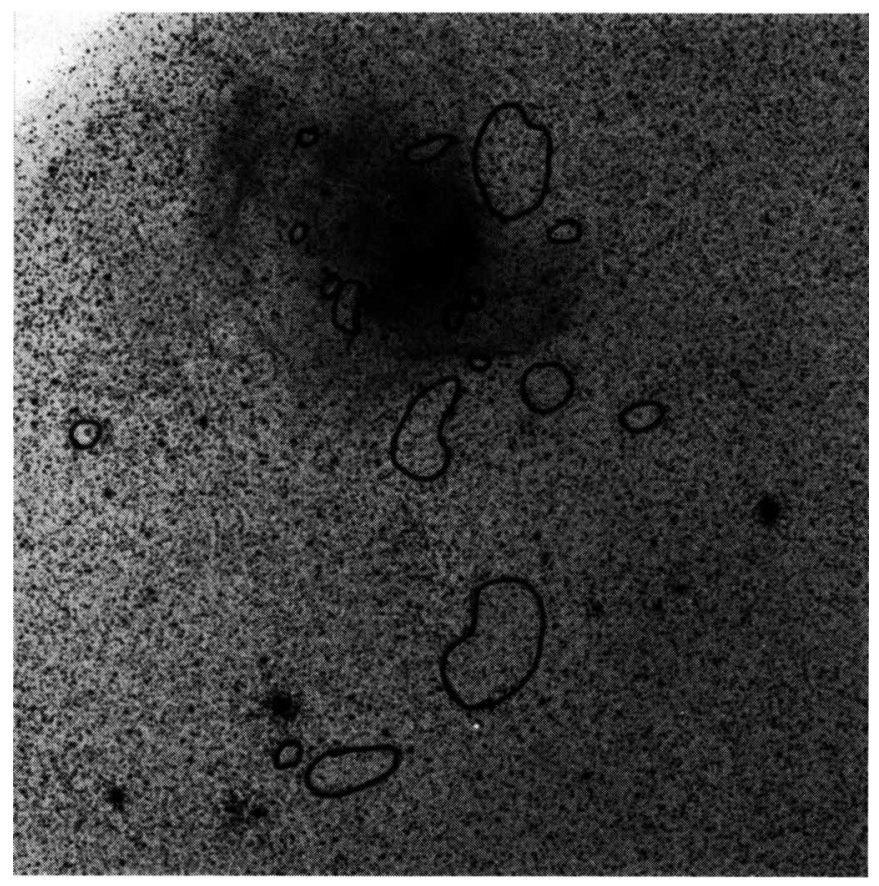

Figure 3. A portion of the SMC from a CTIO 4-m B plate with dark nebulae identified (from Hodge 1990). The large object is NGC 346.

Figure 3 shows a small region of the SMC in which some of the discrete dust clouds are marked. The SMC dark nebulae have somewhat smaller opacities than those in the LMC or the Galaxy, and show a correlation between 
their opacities and their sizes, with the smaller objects having the higher opacities, on the average.

As shown in Hodge (1974b), the dark nebulae of the SMC agree in position remarkably well with the HI distribution (Hindman et al. 1963). The peak density is near the centre of the core, and there is an eastward extension towards the wing in both $\mathrm{HI}$ and dust cloud distributions.

\subsection{THE LMC}

The dark nebulae of the LMC have been charted and discussed by Hodge $(1972,1988)$ and van den Bergh (1974). As for the SMC, the selection by eye has a strong subjective element, both because of the highly diffuse nature of the objects themselves and because of the highly variable nature of the background light. Nevertheless, the searches agree quite well. The 4-m plates tended to turn up many more smaller dust clouds than the Schmidt plates, as should be expected considering the very different resolutions (the scales of the plates are 18.5 and $97 \mathrm{arcsec} / \mathrm{mm}$, respectively). The mean dimensions for those found on the Schmidt plates is $61 \mathrm{pc}$, while the mean for the $4-\mathrm{m}$ plates is $26 \mathrm{pc}$. In either case, the sizes are larger than those of common dark nebulae catalogued in our Galaxy, indicating that what we see in the MCs correspond to the Galaxy's "large dust complexes".

Unlike the case of the SMC, the spatial distribution of the dark nebulae is conspicuously different from that of the HI. Both do show a concentration in the region of 30 Doradus, but the actual centre of the dust cloud distribution is considerably to the west of that location (at $5^{\mathrm{h}} 25^{\mathrm{m}},-69.6^{\circ}$ ).

Comparison can also be made with the distribution of molecular gas (e.g. $\mathrm{CO}$ as mapped by Cohen et al. (1988). In general, there is good agreement with the (lower resolution) $\mathrm{CO}$ contours, but there are also several cases of conspicuous disagreements. For some dark nebulae, there is no evidence of molecular gas, while for some $\mathrm{CO}$ peaks, there are no dark nebulae. To pursue these surprising inconsistencies would be of great interest in connection with questions about the mode of star formation in a low-heavy-element-abundance galaxy. However, to do so properly will require both higher-resolution $\mathrm{CO}$ maps, such as are just now becoming available, and more quantitative optical data, which will be possible soon with large format CCDs.

I am grateful to the National Science Foundation for support through grant AST-8814526 and to the Cerro Tololo Interamerican Observatory for observing time.

\section{References}

Boggs, D.: (1990), PhD thesis, University of Washington, SEattle, WA USA. Clayton, G. \& Martin, P.: (1985), Astrophys. J., 288, 558.

Cohen, R.S, Dame, T.M., Garay, G., Montani, J., Rubio, M., \& Thaddeus, P.: (1988), Astrophys. J., 331, L95.

Fitzpatrick, E.: (1985), Astrophys. J., 299, 219.

Greve, A., van Genderen, A., \& Laval, A.: (1990), IRAM Preprint no. 179.

Gurwell, M. \& Hodge, P.: (1990), Publ. Astron. Soc. Pac., in press.

Hindman, J.V., Kerr, F.J. \& McGee, R.X.: (1963), Aust. J. Phys., 16, 570.

Hodge, P.: (1972), Publ. Astron. Soc. Pac., 84, 365.

Hodge, P.: (1974a), Astrophys. J., 192, 21.

Hodge, P.: (1974b), Publ. Astron. Soc. Pac., 86, 263.

Hodge, P.: (1988), Publ. Astron. Soc. Pac., 100, 346.

Hodge, P.: (1990), 'Visual and automated searches for small discrete dust clouds". Preprint.

Hutchings, J.: (1982), Astrophys. J., 255, 70. 
Isserstedt, J.: (1975), Astron. Astrophys., 41, 175.

Koornneef, J. \& Code, A.: (1981), Astrophys. J., 247, 860.

Koornneef, J.: (1982), Astron. Astrophys., 107, 247.

Lee, M.G.: (1990), PhD thesis, University of Washington, Seattle, WA, USA.

Lequeux, J., Maurice, E. \& Prevot, L.: (1984), in: 'Structure and Evolution of the Magellanic Clouds', (eds S. van den Bergh \& K. de Boer), Reidel,

Dordrecht, pp. 405-406.

MacGillivray, H.: (1975), Mon. Not. R. Astron. Soc., 170, 241.

Morgan, D. \& Nandy, K.: (1982), Mon. Not. R. Astron. Soc., 199, 979.

Nandy, K., Morgan, D., Willis, A., Wilson, R \& Gondhalekar, P.: (1981), Mon. Not. R. Astron. Soc., 196, 955.

Payne-Gaposchkin, C. \& Gaposchkin, S.: (1966), Smithsonian Contrib. Astrophys., 9, 1-202 (whole vol.)

Schwering, P.: (1988), PhD thesis, Universiteit te Leiden.

van den Bergh, S.: (1974), Astrophys. J., 193, 63.

Wesselink, A.: (1961), Mon. Not. R. Astron. Soc., 122, 503. 